\title{
BEURLING-MALLIAVIN THEORY FOR TOEPLITZ KERNELS
}

\author{
N. MAKAROV AND A. POLTORATSKI
}

\begin{abstract}
We consider the family of Toeplitz operators $T_{J \bar{S}^{a}}$ acting in the Hardy space $H^{2}$ in the upper halfplane; $J$ and $S$ are given meromorphic inner functions, and $a$ is a real parameter. In the case where the argument of $S$ has a power law type behavior on the real line, we compute the critical value

$$
c(J, S)=\inf \left\{a: \operatorname{ker} T_{J \bar{S} a} \neq 0\right\} .
$$
\end{abstract}

The formula for $c(J, S)$ generalizes the Beurling-Malliavin theorem on the radius of completeness for a system of exponentials.

\section{InTRODUCTION AND RESUlts}

1.1. Completeness of complex exponentials. For $\Lambda \subset \mathbf{C}$ denote

$$
\mathcal{E}_{\Lambda}=\left\{e^{i \lambda x}: \lambda \in \Lambda\right\} .
$$

By definition, the radius of completeness for the family $\mathcal{E}_{\Lambda}$ is the number

$$
R(\Lambda)=\sup \left\{a: \mathcal{E}_{\Lambda} \text { is complete in } L^{2}(-a, a)\right\} .
$$

(A family is complete if finite linear combinations of its elements are dense in the corresponding space.) In [1]-[2], Beurling and Malliavin established a formula for $R(\Lambda)$ in terms a certain density of $\Lambda$ at infinity.

If $\Lambda \subset \mathbb{R}$, then the effective (or Beurling-Malliavin) density $D_{\text {eff }}(\Lambda)$ is the supremum of the set of numbers $a \geq 0$ such that there is a collection of disjoint intervals $\left\{l_{j}\right\}$ satisfying the following two conditions:

$$
\sum_{j} \frac{\left|l_{j}\right|^{2}}{1+d_{j}^{2}}=\infty, \quad d_{j}:=\operatorname{dist}\left(0, l_{j}\right),
$$

and

$$
\forall j, \quad \#\left(\Lambda \cap l_{j}\right) \geq a\left|l_{j}\right|
$$

Beurling-Malliavin's "Second Theorem" (BM2 for short) states that if $\Lambda \subset \mathbb{R}$, then

$$
R(\Lambda)=\pi D_{\text {eff }}(\Lambda) .
$$

This formula extends to the general case $\Lambda \subset \mathbf{C}$ as follows. If $\Lambda$ satisfies the Blaschke condition

$$
\sum_{\lambda \in \Lambda}\left|\Im \lambda^{-1}\right|<\infty
$$

then

$$
R(\Lambda)=\pi D_{\text {eff }}\left(\Lambda^{*}\right)
$$

The first author is supported by N.S.F. Grant No. 0201893 .

The second author is supported by N.S.F. Grant No. 0500852. 
where

otherwise

$$
\Lambda^{*}=\left\{\lambda^{*}: \lambda \in \Lambda, \Re \lambda \neq 0\right\}, \quad \lambda^{*}:=\left[\Re \lambda^{-1}\right]^{-1} ;
$$

$$
\Lambda \notin(B) \quad \Rightarrow \quad R(\Lambda)=\infty
$$

The Beurling-Malliavin theorem crowned a long search for a solution of the completeness problem, see [29], [21], [32], [20]. We refer to [31] for historical information; let us only mention that one of the earliest results of the theory was the estimate

$$
R(\Lambda) \leq \pi D(\Lambda), \quad(\Lambda \subset \mathbb{R}),
$$

where $D(\Lambda)$ is the usual upper density of $\Lambda$ at infinity.

The Beurling-Malliavin theory also comprises their "First Theorem" (BM1), a result of considerable independent interest and (so far) a necessary step in the proof of BM2. A detailed exposition of BM theory (including clarification and further improvements of the argument) is presented in the monographs [17], [18], [9], see also [14], [6]. New applications and new approaches to various parts of the theory have been suggested; see [3], [10], [24] for some recent developments; also see [15] for a modern overview of the completeness problem for exponential systems.

In this paper we generalize BM theory to many other families of special functions. We state our results in the language of Toeplitz kernels referring to our paper [23] for a detailed explanation of how results of this type are related to the completeness problem for families of solutions of general Sturm-Liouville problems.

1.2. Toeplitz kernels. The completeness radius problem can be restated in terms of Toeplitz operators as follows. Recall that the Toeplitz operator $T_{U}$ with a symbol $U \in L^{\infty}(\mathbb{R})$ is the map

$$
T_{U}: H^{2} \rightarrow H^{2}, \quad F \mapsto P_{+}(U F),
$$

where $P_{+}$is the orthogonal projection in $L^{2}(\mathbb{R})$ onto the Hardy space $H^{2}=H^{2}\left(\mathbf{C}_{+}\right)$ in the upper halfplane $\mathbf{C}_{+}=\{\Im z>0\}$. By duality and the definition of the classical Fourier transform,

$$
f(t) \mapsto \hat{f}(z)=\int e^{i z t} f(t) d t
$$

the exponential family $\mathcal{E}_{\Lambda}$ is complete in $L^{2}(-a, a)$ if and only if there is a non-trivial function $F$ in the Paley-Wiener space

$$
\mathrm{PW}_{a}=\left\{\hat{f}: f \in L^{2}(-a, a)\right\}
$$

such that $F=0$ on $\Lambda$. According to Paley-Wiener's theorem, the Fourier transform isometrically identifies $L^{2}(0, \infty)$ with $H^{2}\left(\mathbf{C}_{+}\right)$, and therefore

$$
\mathrm{PW}_{a}=e^{-i a z}\left[H^{2} \ominus e^{2 a i z} H^{2}\right] .
$$

The subspace $H^{2} \ominus e^{2 a i z} H^{2}$ is the so called model space of the inner function $e^{2 a i z}$. More generally, one defines model spaces

$$
K_{\Theta}=H^{2} \ominus \Theta H^{2}
$$

for arbitrary inner functions $\Theta$. The elements of $K_{\Theta}$ are analytic functions in $\mathbf{C}_{+}$ but if $\Theta$ has a meromorphic extention to the whole complex plane, (we call such $\Theta$ 's 
meromorphic inner functions), then the elements of $K_{\Theta}$ are defined as functions in C. The completeness problem for exponentials is exactly the problem of describing the sets of uniqueness for the model space of $e^{2 a i z}$.

Suppose now that $\Lambda$ is a subset of $\mathbf{C}_{+}$satisfying the Blaschke condition, and let $B_{\Lambda}$ be the corresponding Blaschke product. A simple argument shows that $\Lambda$ is a set of uniqueness for $K_{\Theta}$ if and only if the Toeplitz operator with the symbol $U=B_{\Lambda} \bar{\Theta}$ has a trivial kernel. In particular, we obtain the formula

$$
R(\Lambda)=\inf \left\{a: \operatorname{ker} T_{B_{\Lambda} e^{-2 a i z}} \neq 0\right\} .
$$

There is a similar statement in the general case $\Lambda \subset \mathbf{C}$, see [23], Section 3.1. For example, if $\Lambda \subset \mathbb{R}$, then

$$
R(\Lambda)=\inf \left\{a: \operatorname{ker} T_{J_{\Lambda} e^{-2 a i z}} \neq 0\right\},
$$

where $J_{\Lambda}$ denotes some/any meromorphic inner function $J$ such that $\Lambda$ is precisely the level set $\{J=1\}$.

We should mention that the idea of the Toeplitz operator approach in the study of exponential systems was introduced in the series of papers [30], [26], [12]. This approach has been particularly successful for the interpolation and sampling theory in Paley-Wiener spaces, see [22], [28], [33].

We will use the following notation for kernels of Toeplitz operators (or Toeplitz kernels in $H^{2}$ ):

$$
N[U]=\operatorname{ker} T_{U} .
$$

(For example, $N[\bar{\Theta}]=K_{\Theta}$ if $\Theta$ is an inner function.) We will also consider Toeplitz kernels in the Smirnov-Nevanlinna class $\mathcal{N}^{+}=\mathcal{N}^{+}\left(\mathbf{C}_{+}\right)$,

$$
N^{+}[U]=\left\{F \in \mathcal{N}^{+} \cap L_{\text {loc }}^{1}(\mathbb{R}): \bar{U} \bar{F} \in \mathcal{N}^{+}\right\},
$$

and in the Hardy spaces $H^{p}=H^{p}\left(\mathbf{C}_{+}\right)$,

$$
N^{p}[U]=N^{+}[U] \cap L^{p}(\mathbb{R}), \quad(0<p \leq \infty) .
$$

See [16], [7], [27] for general references concerning the Hardy-Nevanlinna theory.

1.3. Generalization of Beurling-Malliavin theory. A natural way to generalize the completeness radius problem (and the BM2 theorem) is to ask about the exact value of the infimum

$$
\inf \left\{a: \operatorname{ker} T_{J \bar{S}^{a}} \neq 0\right\}
$$

for arbitrary meromorphic inner functions $J$ and $S$. We will give an answer in the case where the argument of $S$ has a power law type behavior,

$$
(\arg S)^{\prime}(x) \asymp|x|^{\kappa}, \quad x \rightarrow \pm \infty,
$$

with $\kappa \geq 0$. (We call the case $\kappa \geq 0$ super-exponential to underline the relation to the classical case $S(x)=e^{i a x}$. In Section 1.5 below we will comment on the sub-exponential case $\kappa<0$.)

As explained in [23], the computation of the "radius" (1.2) has some immediate consequences for the theory of Sturm-Liouville (SL) operators. Roughly speaking, the case of SL operators with eigenvalues

$$
\lambda_{n} \asymp n^{\nu}
$$


belongs to the theory with parameter

$$
\kappa=\frac{2}{\nu}-1 \geq 0 .
$$

If $\kappa>0$, the SL operators are singular in contrast to the BM case $S(x)=e^{i a x}$, which applies to regular operators. In addition to the completeness problem for systems of solutions of SL equations, cf [11], the generalized BM theory applies to certain problems of spectral theory as well as the theory of (Weyl-Titchmarsh) Fourier transforms associated with SL operators and the corresponding (de Branges) spaces of entire functions.

To state our results, we need to introduce the notion of $B M$ intervals. Let $\gamma$ be a continuous function $\mathbb{R} \rightarrow \mathbb{R}$ such that $\gamma(\mp \infty)= \pm \infty$. i.e.

$$
\lim _{x \rightarrow-\infty} \gamma(x)=+\infty, \quad \lim _{x \rightarrow+\infty} \gamma(x)=-\infty .
$$

The family $\mathcal{B M}(\gamma)$ is defined as the collection of the components of the open set

$$
\left\{x: \gamma(x) \neq \max _{[x,+\infty)} \gamma\right\} \text {. }
$$

For an interval $l \in \mathcal{B M}(\gamma)$, we denote its length by $|l|$ or simply by $l$, and we denote the distance to the origin by $d=d(l)$.

1.4. Super-exponential case. If $\kappa \geq 0$, then we say that $\gamma$ is $(\kappa)$-almost decreasing if

$$
\gamma(\mp \infty)= \pm \infty, \quad \sum_{l \in \mathcal{B M}(\gamma)} d^{\kappa-2} l^{2}<\infty
$$

where the sum is taken over intervals satisfying $d(l) \geq 1$.

The standard terminology in the classical $\kappa=0$ case is the following: the family $\mathcal{B M}(\gamma)$ is short if $\gamma$ is almost decreasing; otherwise we say that $\mathcal{B M}(\gamma)$ is long.

Theorem A. Let $\kappa \geq 0$, and let $U=e^{i \gamma}$ and $S=e^{i \sigma}$ be smooth unimodular functions on $\mathbb{R}$ such that

$$
\gamma^{\prime}(x) \gtrsim-|x|^{\kappa}, \quad \sigma^{\prime}(x) \gtrsim|x|^{\kappa}, \quad(x \rightarrow \infty) .
$$

(i) If $\gamma$ is not $(\kappa)$-almost decreasing, then $N^{+}\left[U S^{\epsilon}\right]=0$ for all $\epsilon>0$.

(ii) If $\gamma$ is $(\kappa)$-almost decreasing, then $N^{p}\left[U \bar{S}^{\epsilon}\right] \neq 0$ for all $\epsilon>0$ and all $p<\frac{1}{3}$.

Here and throughout the paper the notation $f(x) \gtrsim g(x)$ means that $f(x) \geq c g(x)$ for some $c>0$ and all $x$ such that $|x| \gg 1$.

Given two unimodular functions $U$ and $S$ as in Theorem A, we can consider the family of symbols

$$
U \bar{S}^{a}=e^{i \gamma_{a}}, \quad \gamma_{a}=\gamma-a \sigma, \quad(a \in \mathbb{R}) .
$$

If $a_{1}>a$ and if $\gamma_{a}$ is decreasing near $\infty$, then $\gamma_{a_{1}}$ is also decreasing. It is not difficult to see that the same is true for almost decreasing functions, so we can define the transition parameter

$$
c \equiv c(U, S ; \kappa)=\inf \left\{a: \gamma_{a} \text { is }(\kappa) \text {-almost descreasing }\right\} \in(-\infty,+\infty] .
$$


Corollary. Let $U=e^{i \gamma}$ and $S=e^{i \sigma}$ be such that

$$
\gamma^{\prime}(x) \gtrsim-|x|^{\kappa}, \quad \sigma^{\prime}(x) \asymp|x|^{\kappa}, \quad(x \rightarrow \infty),
$$

and let $c=c(U, S ; \kappa)$. Then for all $p<1 / 3$ we have

$$
N^{p}\left[U \bar{S}^{a}\right]=0 \quad(a<c), \quad N^{p}\left[U \bar{S}^{a}\right] \neq 0 \quad(a>c) .
$$

Indeed, if $a<c$ then $\gamma_{a+\epsilon}$ is not almost decreasing for some $\epsilon>0$, and we have

$$
N^{p}\left[U \bar{S}^{a}\right] \subset N^{+}\left[U \bar{S}^{a+\epsilon} S^{\epsilon}\right]=0
$$

by Theorem A, which can be applied because $\gamma_{a}^{\prime}(x) \gtrsim-|x|^{\kappa}$ for all $a$ 's. Similarly, if $a>c$, then $\gamma_{a-\epsilon}$ is almost decreasing for some $\epsilon>0$, and we have

$$
N^{p}\left[U \bar{S}^{a}\right]=N^{p}\left[U \bar{S}^{a-\epsilon} \bar{S}^{\epsilon}\right] \neq 0 .
$$

In the special case where $U$ is an inner function, we can extend the statement of he corollary to all values of $p$, in particular $p=2$.

Theorem B. Let $J$ be a meromorphic inner function, and suppose that a unimodular function $S$ satisfies

$$
(\arg S)^{\prime}(x) \asymp|x|^{\kappa}, \quad x \rightarrow \infty .
$$

Denote $c=c(J, S ; \kappa)$. Then for all $p \leq \infty$ we have

$$
N^{p}\left[J \bar{S}^{a}\right]=0 \quad(a<c), \quad N^{p}\left[J \bar{S}^{a}\right] \neq 0 \quad(a>c) .
$$

1.5. Sub-exponential case. It is easy to see that the statement of Theorem A (and Theorem B) can not be extended to the case $\kappa<0$. For example, the functions

$$
\sigma(x)=2 \operatorname{sign}(x)|x|^{1 / 4}, \quad \gamma(x)=2(1+\sqrt{2}) 1_{\mathbb{R}_{-}}(x)|x|^{1 / 4}
$$

satisfy the conditions (1.4) with $\kappa=-3 / 4$, and of course $\gamma(+\infty) \neq-\infty$. Nevertheless, for $U=e^{i \gamma}$ and $S=e^{i \sigma}$ we have

$$
N^{\infty}[U S] \neq 0,
$$

because

$$
U S=\bar{f} / f, \quad f(z)=\exp \left\{-(1+i) z^{1 / 4}\right\} \in H^{\infty}\left(\mathbf{C}_{+}\right) .
$$

(Also note that the sum $\sum d^{\kappa-2} l^{2}$ in $(1.3)$ is always finite if $\kappa<0$.)

The Beurling-Malliavin theory extends to the sub-exponential case in a different fashion. For $\kappa \in(-1,0]$ we consider the weighted (non-linear) Smirnov-Nevanlinna classes

$$
\mathcal{N}_{\kappa}^{+}=\left\{F \in \mathcal{N}^{+}: \log |F| \in L^{1}\left(\mathbb{R}, \frac{1}{1+|x|^{2+\kappa}}\right)\right\},
$$

and define the corresponding Toeplitz kernels as follows:

$$
N_{\kappa}^{+}[U]=N^{+}[U] \cap \mathcal{N}_{\kappa}^{+}, \quad N_{\kappa}^{p}[U]=N^{p}[U] \cap \mathcal{N}_{\kappa}^{+} .
$$


Theorem C. Let $\kappa \in(-1,0]$, and let $U=e^{i \gamma}$ and $S=e^{i \sigma}$ be smooth unimodular functions such that

$$
\gamma^{\prime}(x) \gtrsim-|x|^{\kappa}, \quad \sigma^{\prime}(x) \gtrsim|x|^{\kappa} \quad(x \rightarrow \infty) .
$$

(i) If the family $\mathcal{B M}(\gamma)$ is long, then $N_{\kappa}^{+}\left[U S^{\epsilon}\right]=0$ for all $\epsilon>0$.

(ii) If the family $\mathcal{B M}(\gamma)$ is short, then $N_{\kappa}^{p}\left[U \bar{S}^{\epsilon}\right] \neq 0$ for all $\epsilon>0$ and all $p<\frac{1}{3}$.

One can also state a theorem similar to Theorem B. Applications of these results to Volterra operators, see [8], and higher order differential operators will be discussed in a separate paper.

1.6. Hilbert transform. The main tool in the proof of the theorems stated above is the one-dimensional Hilbert transform. Let $\Pi$ denote the Poisson measure on $\mathbb{R}$,

$$
d \Pi(t)=\frac{d t}{1+t^{2}} .
$$

If $h \in L_{\Pi}^{1} \equiv L^{1}(\mathbb{R}, \Pi)$ is a real-valued function, and if $\mathcal{S} h$ denotes its Schwarz integral,

$$
\mathcal{S h}(z)=\frac{1}{\pi i} \int_{\mathbb{R}}\left[\frac{1}{t-z}-\frac{t}{1+t^{2}}\right] h(t) d t, \quad\left(z \in \mathbf{C}_{+}\right),
$$

then $\tilde{h}$, the Hilbert transform of $h$, is defined a.e. on $\mathbb{R}$ as the angular limit of $\Im[\mathcal{S} h]$. Alternatively, $\tilde{h}$ can be defined as a singular integral:

$$
\tilde{h}(x)=\frac{1}{\pi} \text { v.p. } \int\left[\frac{1}{x-t}+\frac{t}{1+t^{2}}\right] h(t) d t, \quad(x \in \mathbb{R}) .
$$

(As a general rule we identify Nevanlinna class functions in the halfplane $\mathbf{C}_{+}$with their angular boundary values on $\mathbb{R}$; e.g. we may write $\mathcal{S} h=h+i \tilde{h}$.)

The relevance of the Hilbert transform in the theory of Toeplitz kernels can be explained by the following simple observation, see [23], Section 2 .

Suppose $\gamma: \mathbb{R} \rightarrow \mathbb{R}$ is a smooth function. Then $N^{+}\left[e^{i \gamma}\right] \neq 0$ if and only if

$$
\gamma=-\alpha+\tilde{h}
$$

for some smooth increasing function $\alpha$ and some $h \in L_{\Pi}^{1}$. There is a similar criterion for Toeplitz kernels in Hardy spaces: $N^{p}\left[e^{i \gamma}\right] \neq 0$ if and only if $\gamma$ admits a representation (1.6) with $\alpha$ being the argument of some inner function and with $h \in L_{\Pi}^{1}$ such that $e^{-h} \in L^{p / 2}(\mathbb{R})$.

For further references, we recall some properties of the Hilbert transform. We denote by $L_{\Pi}^{(1, \infty)}$ the usual weak $L^{1}$-space with respect to the Poisson measure. Kolmogorov's theorem states that

$$
\tilde{h} \in L_{\Pi}^{o(1, \infty)},
$$

where $L_{\Pi}^{o(1, \infty)}$ stands for the "little o" subspace of $L_{\Pi}^{(1, \infty)}$, i.e.

$$
\Pi\{|\tilde{h}|>A\}=o\left(\frac{1}{A}\right), \quad A \rightarrow \infty .
$$


For bounded functions we have the following (Smirnov-Kolmogorov) estimate :

$$
\|h\|_{\infty}<\frac{\pi}{2} \quad \Rightarrow \quad e^{\tilde{h}} \in L_{\Pi}^{1} .
$$

Together with the criterion (1.6), this implies

$$
\|\gamma\|_{\infty}<\frac{\pi}{p} \quad \Rightarrow \quad N^{p}\left[\bar{b}^{2 / p} e^{i \gamma}\right] \neq 0
$$

where $b$ is the Blaschke factor

$$
b(z)=\frac{i-z}{i+z}, \quad z \in \mathbf{C}_{+} .
$$

1.7. The structure of BM theory. In the remaining sections of the paper we prove Theorems A and B. We closely follow all the steps in our presentation of the classical Beurling-Malliavin theory in [23], combining them with certain estimates of the Hilbert transform, which we derive in Section 2. To make the proof selfcontained, in several places we had to repeat the argument outlined in [23]. To avoid further repetitions we decided to omit the proof of Theorem $\mathrm{C}$ because the reasoning in the sub-exponential case is quite similar. The proof of Theorems A and $\mathrm{B}$ is organized as follows.

(1) Upper density estimate: $\gamma( \pm \infty) \neq \mp \infty$ implies $N^{+}\left[U S^{\epsilon}\right]=0$; Section 3.1.

This statement is analogous to the estimate (1.1).

(2) Effective density estimate: $\sum d^{\kappa-2} l^{2}=\infty$ implies $N^{+}\left[U S^{\epsilon}\right]=0$; Sections 3.2-

3.4. Together with (1) this generalizes the estimate $R(\Lambda) \leq \pi D_{\text {eff }}(\Lambda)$ in BM2.

(3) Little multiplier theorem: if $\gamma$ is almost decreasing, then $N^{+}\left[U \bar{S}^{\epsilon}\right]=0$; Section 4 .

(4) BM multiplier theorem: if the weighted Dirichlet norm of $\log W$ is finite, then $W$ belongs to some Hardy space up to a factor from $N^{+}\left[\bar{S}^{\epsilon}\right]$; Sections 5.1-5.2.

(5) A version of BM1: the logarithm of any outer function in $\mathrm{N}^{+}$-kernel has a finite weighted Dirichlet norm. This is used to show that non-triviality of $N^{+}$-kernels implies non-triviality of $N^{p}$-kernels for symbols involving inner functions; Sections 5.3-5.4.

(6) $L^{p}$-multipliers: approximation by inner functions and multiplying the elements of $N^{p}$-kernels down to $H^{\infty}$; Section 6 .

\section{ONE-SIDED LIPSChITZ CONDITION FOR THE HilBeRT TRANSFORM}

In this section we discuss various consequences of the weighted one-sided Lipschitz condition

$$
\tilde{h}^{\prime}(x) \lesssim|x|^{\kappa}, \quad x \rightarrow \infty,
$$

for smooth real-valued functions $h \in L_{\Pi}^{1}$. (Here and elsewhere $\tilde{h}^{\prime}$ means $(\tilde{h})^{\prime}$.) 


\subsection{Application of Kolmogorov's theorem.}

Lemma 1. If $\kappa \geq 0$ and $h \in L_{\Pi}^{1}$, then

$$
\tilde{h}^{\prime}(x) \lesssim x^{\kappa} \quad \Rightarrow \quad \tilde{h}(x)=o\left(x^{\kappa+1}\right) \quad \text { as } \quad x \rightarrow+\infty .
$$

Proof: By Kolmogorov's theorem, we have

$$
\tilde{h} \in L_{\Pi}^{o(1, \infty)} .
$$

If $x_{*} \gg 1$ and $\tilde{h}\left(x_{*}\right) \geq c x_{*}^{\kappa+1}$ for some $c>0$, then for all $x$ such that $1 \ll x \leq x_{*}$ we have

$$
\tilde{h}(x) \geq \tilde{h}\left(x_{*}\right)-a \int_{x}^{x_{*}} t^{\kappa} d t \geq c x_{*}^{\kappa+1}-a\left(x_{*}^{\kappa+1}-x^{\kappa+1}\right),
$$

and it follows that

$$
\tilde{h}(x) \gtrsim x_{*}^{\kappa+1} \geq x_{*}
$$

for all $x$ in some interval $\left(x_{* *}, x_{*}\right)$ of length $\asymp x_{*}$. Since $\Pi\left(x_{* *}, x_{*}\right) \asymp 1 / x_{*}$, this contradicts (2.1), see (1.7).

If $\tilde{h}\left(x_{*}\right) \leq-c x_{*}^{\kappa+1}$ for some $c>0$, then by a similar argument we have

$$
\tilde{h}(x) \lesssim-x_{*}^{\kappa+1} \leq x_{*}
$$

for all $x$ in some interval $\left(x_{*}, x_{* *}\right)$ of length $\asymp x_{*}$, which again contradicts $(2.1)$.

We will also need the following version of this lemma.

Lemma 2. Let $h \in L_{\Pi}^{1}, \kappa \geq 0$, and $a \in \mathbb{R}$. If

$$
\tilde{h}^{\prime}(x)+a x^{-1} \tilde{h}(x) \leq x^{\kappa}, \quad x \gg 1,
$$

then

$$
\tilde{h}(x)=o\left(x^{\kappa+1}\right) \quad \text { and } \quad \tilde{h}^{\prime}(x) \leq x^{\kappa}+o\left(x^{\kappa}\right) \quad \text { as } \quad x \rightarrow+\infty .
$$

Proof: Suppose we have $\tilde{h}\left(x_{*}\right) \geq c x_{*}^{\kappa+1}$ for some $x_{*} \gg 1$. Let $x_{1}$ be the smallest positive number such that $\tilde{h}\left(x_{1}\right)=c x_{*}^{\kappa+1}$, so we have $1 \ll x_{1} \leq x_{*}$ and $\tilde{h} \leq c x_{*}^{\kappa+1}$ on $\left(0, x_{1}\right)$. Together with $(2.2)$, this implies

$$
\tilde{h}^{\prime}(x) \lesssim \frac{x_{*}^{\kappa+1}}{x_{1}}, \quad x \in\left(\frac{x_{1}}{2}, x_{1}\right) .
$$

Arguing as in the previous proof, we see that $\tilde{h} \gtrsim x_{*}^{\kappa+1} \geq x_{1}$ on some interval of length $\asymp x_{1}$, which contradicts the weak $L^{1}$-estimate (1.7). The argument in the case $\tilde{h}\left(x_{*}\right) \leq-c x_{*}^{\kappa+1}$ is similar. 


\subsection{Hilbert transform in weighted $L^{1}$-spaces.}

Lemma 3. If $\kappa \in[-1,0)$ and $h \in L^{1}\left(|x|^{-2-\kappa}\right)$, then

$$
\tilde{h}^{\prime}(x) \lesssim x^{\kappa} \Rightarrow \tilde{h}(x)=o\left(x^{\kappa+1}\right), \quad x \rightarrow+\infty .
$$

Proof: If $\kappa \in(-1,0)$, then the weight $|x|^{-2-\kappa}$ satisfies the Muckenhoupt $\left(A_{1}\right)$ condition at infinity, and therefore we have

$$
h \in L^{1}\left(|x|^{-2-\kappa}\right) \quad \Rightarrow \quad \tilde{h} \in L^{o(1, \infty)}\left(|x|^{-2-\kappa}\right),
$$

see [13]. (One can also give an elementary proof for this particular weight.) We then argue as in the proof of Lemma 1. For example, if $x_{*} \gg 1$ and $\tilde{h}\left(x_{*}\right) \geq c x_{*}^{\kappa+1}$, then $\tilde{h} \gtrsim x_{*}^{\kappa+1}$ on some interval $\left[x_{* *}, x_{*}\right]$ of length $\asymp x_{*}$. The weighted length of this interval is $\asymp x_{*}^{-1-\kappa}$, which contradicts the weak $L^{1}$-estimate.

If $\kappa=-1$, then we consider the function

$$
h_{1}(x)=x h(x) \in L_{\Pi}^{1} .
$$

Since $\tilde{h}_{1}(x)=x \tilde{h}(x)$, we have

$$
\tilde{h}_{1}^{\prime}(x)=\tilde{h}(x)+x \tilde{h}^{\prime}(x) \leq x^{-1} \tilde{h}_{1}(x)+O(1), \quad x \rightarrow+\infty .
$$

By Lemma 2, we get $\tilde{h}_{1}(x)=o(x)$ and therefore $\tilde{h}(x)=o(1)$.

\subsection{Persistence of 1-sided Lipschitz condition.}

Lemma 4. Let $f \in L_{\Pi}^{1}, 0 \notin \operatorname{supp} f$, and let

$$
0 \leq \alpha \leq \beta, \quad \text { or } \quad 0 \leq \beta<\alpha<2 \text {. }
$$

Denote

$$
g(x)=|x|^{-\alpha} f(x)
$$

Then

$$
\tilde{f}^{\prime}(x) \leq(1+o(1))|x|^{\beta} \Rightarrow \tilde{g}^{\prime}(x) \leq(1+o(1))|x|^{\beta-\alpha},
$$

and

$$
x^{-1} \tilde{f}^{\prime}(x) \leq(1+o(1))|x|^{\beta-1} \Rightarrow x^{-1} \tilde{g}^{\prime}(x) \leq(1+o(1))|x|^{\beta-\alpha-1} .
$$

Proof: We will prove (2.3) for $x \rightarrow+\infty$. The proof of the other cases is similar. Since the statement is trivial for $\alpha=0$, we will assume $\alpha>0$.

It is clear that we can modify $f$ on any finite interval, so we will assume that $f(x)=x^{N}$ near the origin for some $N \gg 1$. If we specify $\tilde{f}(0)=0$, then

$$
|x|^{-\alpha} \tilde{f}(x) \in L_{\Pi}^{1} \text {. }
$$

Indeed, by Lemma 1 we have $\tilde{f}=O\left(|x|^{N}\right)$, and therefore

$$
|x|^{-\alpha}|\tilde{f}|=|x|^{-\alpha}|\tilde{f}|^{\alpha / N}|\tilde{f}|^{1-(\alpha / N)} \lesssim|\tilde{f}|^{1-(\alpha / N)} \in L_{\Pi}^{1}
$$

by Kolmogorov's estimate (1.7).

Consider the analytic function

$$
u(z)+i \tilde{u}(z):=z_{9}^{-\alpha}(f+i \tilde{f})(z), \quad z \in \mathbf{C}^{+},
$$


where $z^{-\alpha}$ denotes the branch positive on $\mathbb{R}_{+}$. Note that

$$
u(x)=g(x), \quad \tilde{u}(x)=|x|^{-\alpha} \tilde{f}(x) \quad \text { for } \quad x \in \mathbb{R}_{+},
$$

and

$$
u(x)=|x|^{-\alpha}[f(x) \cos \alpha \pi+\tilde{f}(x) \sin \alpha \pi] \quad \text { for } \quad x \in \mathbb{R}_{-} .
$$

By $(2.5)$,

$$
g-u \in L_{\Pi}^{1}, \quad g-u=0 \quad \text { on } \quad \mathbb{R}_{+},
$$

so if we define

$$
\delta(x)=\tilde{g}(x)-|x|^{-\alpha} \tilde{f}(x),
$$

then $\delta=\tilde{g}-\tilde{u}$ on $\mathbb{R}_{+}$, and we have the following representation for the derivative:

$$
\delta^{\prime}(x)=\widetilde{(g-u}^{\prime}(x)=\int_{-\infty}^{0} \frac{c_{1} f(t)+c_{2} \tilde{f}(t)}{(t-x)^{2}} \frac{d t}{|t|^{\alpha}}, \quad(x>0) .
$$

By the dominated convergence theorem

$$
\delta^{\prime}(x)=o(1), \quad x \rightarrow+\infty,
$$

in particular

$$
\delta^{\prime}(x)=o\left(x^{\beta-\alpha}\right) \quad \text { if } \quad \beta \geq \alpha .
$$

In the case $0 \leq \beta<\alpha<2$, we consider the integrals involving $f$ and $\tilde{f}$ separately. We have

$$
\begin{aligned}
\int_{-\infty}^{-1} \frac{|f(t)|}{(t-x)^{2}} \frac{d t}{|t|^{\alpha}} & \leq \int_{-\infty}^{-x} \frac{1}{|t|^{\alpha}} \frac{|f(t)| d t}{|t|^{2}}+\frac{1}{x^{\alpha}} \int_{-x}^{-1} \frac{|t|^{2-\alpha}}{x^{2-\alpha}} \frac{|f(t)| d t}{|t|^{2}} \\
& \leq \frac{1}{x^{\alpha}} \int_{-\infty}^{-x} \frac{|f(t)| d t}{|t|^{2}}+\frac{1}{x^{\alpha}} \int_{-x}^{-1} \frac{|f(t)| d t}{|t|^{2}}=o\left(x^{-\alpha}\right)=o\left(x^{\beta-\alpha}\right) .
\end{aligned}
$$

Since $\beta \geq 0$, by Lemma 1 we have

$$
\tilde{f}(t)=o\left(|t|^{1+\beta}\right),
$$

and since

$$
\int_{-\infty}^{-1} \frac{|t|^{1+\beta-\alpha}}{(t-x)^{2}} d t \leq \int_{-\infty}^{-x}|t|^{\beta-\alpha-1} d t+\frac{1}{x^{2}} \int_{-x}^{-1}|t|^{1+\beta-\alpha} d t \asymp x^{\beta-\alpha},
$$

we have

$$
\int_{-\infty}^{-1} \frac{|\tilde{f}(t)|}{(t-x)^{2}} \frac{d t}{|t|^{\alpha}}=o\left(x^{\beta-\alpha}\right) .
$$

It follows that in all cases we have

$$
\delta^{\prime}(x)=o\left(x^{\beta-\alpha}\right), \quad x \rightarrow+\infty,
$$

and therefore

$$
\tilde{g}^{\prime}(x)=x^{-\alpha} \tilde{f}^{\prime}(x)-\alpha x^{-\alpha-1} \tilde{f}(x)+\delta^{\prime}(x) \leq x^{\beta-\alpha}+o\left(x^{\beta-\alpha}\right) .
$$


2.4. A converse. We will also need a converse of (2.3). We state it only for the range of parameters that will be used later.

Lemma 5. Let $w \in L_{\Pi}^{1}, 0 \notin \operatorname{supp} w$, and

$$
0<\alpha \leq \beta, \quad \text { or } \quad 1 \leq \alpha \leq \min (2, \beta+1) .
$$

Denote

$$
h(x)=|x|^{-\alpha} w(x) .
$$

Then

$$
\tilde{h}^{\prime}(x) \leq(1+o(1))|x|^{\beta-\alpha} \quad \Rightarrow \quad \tilde{w}^{\prime}(x) \leq(1+o(1))|x|^{\beta} .
$$

Proof: (a) The case $\beta \geq \alpha$. Let $n$ be an even integer such that

$$
\alpha_{1}:=n-\alpha \in[0,2) .
$$

Define

$$
g(x):=x^{-n} w(x)=|x|^{-\alpha_{1}} h(x) .
$$

Since $\alpha_{1}<2$ and $\beta_{1}:=\beta-\alpha \geq 0$, we can apply Lemma 4 to $f=h$ and $g$ and obtain the estimate

$$
\tilde{g}^{\prime}(x) \leq|x|^{\beta_{1}-\alpha_{1}}+\cdots=|x|^{\beta-n}+\ldots
$$

Since $\tilde{w}(x)=x^{n} \tilde{g}(x)$, we have

$$
\tilde{w}^{\prime}(x)=n x^{n-1} \tilde{g}(x)+x^{n} \tilde{g}^{\prime}(x) \leq n x^{-1} \tilde{w}(x)+|x|^{\beta}+\ldots,
$$

and by Lemma 2,

$$
\tilde{w}^{\prime}(x) \leq(1+o(1))|x|^{\beta} .
$$

(b) The case $\alpha \in[1,2]$ and $\beta-\alpha \in[-1,0]$. Note that this implies $\beta \geq 0$. Define the functions

$$
g(x)=x^{-1} w(x), \quad f(x)=x h(x),
$$

so

$$
g(x)=|x|^{-\alpha_{1}} f(x), \quad \alpha_{1}=2-\alpha \in[0,1] .
$$

Let us show that

$$
x^{-1} \tilde{f}^{\prime} \leq|x|^{\beta_{1}-1}+\ldots, \quad \beta_{1}:=\beta-\alpha+1 .
$$

Since

$$
h \in L^{1}\left(\frac{1}{|x|^{2-\alpha}}\right) \subset L^{1}\left(\frac{1}{|x|^{2+\kappa}}\right), \quad \kappa:=\beta-\alpha,
$$

by Lemma 3 we have

$$
\tilde{h}(x)=o\left(|x|^{\kappa+1}\right),
$$

and since $\tilde{f}(x)=x \tilde{h}(x)$, we obtain $(2.6)$ :

$$
x^{-1} \tilde{f}^{\prime}(x)=\tilde{h}^{\prime}(x)+x^{-1} \tilde{h}(x) \leq|x|^{\kappa}+o\left(|x|^{\kappa}\right) .
$$

We can now apply Lemma 4 with parameters $\alpha_{1}$ and $\beta_{1}$. (Note that $f \in L_{\Pi}^{1}$ and the parameters are admissible.) By (2.4) we get the estimate

$$
x^{-1} \tilde{g}^{\prime}(x) \leq|x|^{\beta_{1}-\alpha_{1}-1}+\cdots=|x|^{\beta-2}+\ldots,
$$

and from $\tilde{w}(x)=x \tilde{g}(x)$ we derive

$$
\tilde{w}^{\prime}(x)=\tilde{g}(x)+x \tilde{g}^{\prime}(x) \leq x^{-1} \tilde{w}(x)+|x|^{\beta}+\ldots .
$$

Applying Lemma 2 we conclude the proof. 


\section{Triviality of TOePlitz Kernels}

In this section we prove the first part of Theorem $A$, which gives a sufficient condition for the triviality of a Toeplitz kernel. Let us fix $\kappa \geq 0$ and consider two unimodular functions $U=e^{i \gamma}$ and $S=e^{i \sigma}$ on $\mathbb{R}$ satisfying

$$
\gamma^{\prime}(x) \gtrsim-|x|^{\kappa}, \quad \sigma^{\prime}(x) \gtrsim|x|^{\kappa}, \quad(x \rightarrow \infty) .
$$

\subsection{Upper density estimate.}

Proposition. If $N^{+}\left[U S^{\epsilon}\right] \neq 0$ for some $\epsilon>0$, then $\gamma(\mp \infty)= \pm \infty$.

Proof: If $N^{+}\left[U S^{\epsilon}\right] \neq 0$, then by the basic criterion (1.6) we have

$$
\gamma+\epsilon \sigma+\alpha=\tilde{h}, \quad \alpha^{\prime} \geq 0, \quad h \in L_{\Pi}^{1} .
$$

Therefore,

$$
\tilde{h}^{\prime}(x) \gtrsim \gamma^{\prime}(x) \gtrsim-|x|^{\kappa},
$$

and $\tilde{h}(x)=o\left(|x|^{\kappa+1}\right)$ by Lemma 1 . It follows that

$$
\frac{\gamma(x)}{x} \lesssim \frac{\gamma(x)}{x}+\frac{\alpha(x)}{x}=-\epsilon \frac{\sigma(x)}{x}+o\left(|x|^{\kappa}\right) \lesssim-|x|^{\kappa},
$$

which implies $\gamma(\mp \infty)= \pm \infty$.

3.2. Effective density estimate. Let $c>0$ be a fixed constant. For an interval $l \subset \mathbb{R}$ we denote by $l^{\prime}$ and $l^{\prime \prime}$ the intervals of length $c|l|$ adjacent to $l$ from the left and from the right respectively, and we define

$$
\Delta_{l}^{*}[\gamma]=\inf _{l^{\prime \prime}} \gamma-\sup _{l^{\prime}} \gamma
$$

Lemma. Let $\epsilon>0$ and suppose

$$
\gamma(\mp)= \pm \infty, \quad \sum_{l \in \mathcal{B M}(\gamma)} d^{\kappa-2} l^{2}=\infty .
$$

Then there is a constant $c>0$ and there is a collection of disjoint intervals $\left\{l_{n}\right\}$ in $[1,+\infty)$ or in $(-\infty,-1]$ such that

$$
\sum d_{n}^{\kappa-2} l_{n}^{2}=\infty, \quad 10 l_{n} \leq d_{n}, \quad \operatorname{mult}\left\{5 l_{n}\right\}<\infty,
$$

and

$$
\Delta_{l_{n}}^{*}\left[\arg \left(U S^{\epsilon}\right)\right] \geq c d_{n}^{\kappa} l_{n} .
$$

Here $5 l$ is the notation for the interval of length $5|l|$ concentric with $l$, and mult $\{\cdot\}$ is the multiplicity of the covering.

Proof: Suppose the sum (3.2) over BM intervals in $\mathbb{R}_{+}$is infinite. If there are infinitely many BM intervals $l=\left(\tilde{a}_{n}, b_{n}\right)$ in $\mathbb{R}_{+}$satisfying $10|l|>d$, then we set

$$
l_{n}=\left(a_{n}, b_{n}\right), \quad a_{n}:=\frac{10}{11} b_{n} ;
$$


otherwise we simply enumerate BM intervals such that $10|l| \leq d$. In any case, we get a collection of intervals $l_{n}=\left(a_{n}, b_{n}\right)$ satisfying the first two conditions in (3.3) and also the inequality

$$
\gamma\left(b_{n}\right) \geq \gamma\left(a_{n}\right) .
$$

By (3.1), the latter implies that the intervals also satisfy (3.4) for some $c>0$. Finally, we take a subfamily $\left\{l_{n_{k}}\right\}$ such that $\left\{5 l_{n_{k}}\right\}$ is a subcover of $\bigcup 5 l_{n}$ of finite multiplicity and observe that we still have the divergence of the series $\sum d^{\kappa-2} l^{2}$. Indeed, if $\bigcup l_{j} \subset 5 l$, then

and so

$$
d_{j} \asymp d, \quad \sum l_{j}^{2} \lesssim l^{2},
$$

$$
\sum_{j} d_{j}^{\kappa-2} l_{j}^{2} \lesssim d^{\kappa-2} l^{2}
$$

The following proposition completes the proof of the first part of Theorem A.

Proposition. Suppose $\gamma^{\prime}(x) \gtrsim-|x|^{\kappa}$ and suppose there is a collection $\{l\}$ of disjoint intervals in $[1,+\infty)$ such that

$$
\forall l, \quad \Delta_{l}^{*}[\gamma] \geq c l d^{\kappa},
$$

and

$$
\sum d^{\kappa-2} l^{2}=\infty, \quad 10 l \leq d, \quad \operatorname{mult}\{51\}<\infty,
$$

then $N^{+}\left[e^{i \gamma}\right]=0$.

3.3. Proof of the proposition. The statement corresponds to the so-called Beurling's lemma in the classical BM theory. There are several versions of the proof of Beurling's lemma, e.g. Koosis [17] applies the Beurling-Tsuji estimate of harmonic measure, Nazarov [25] uses the Bellman function, and Kargaev's proof [19] is based on PDE techniques. We suggest yet another approach.

According to the criterion (1.6), we have to exclude the possibility

$$
\gamma+\alpha=\tilde{h}, \quad \alpha \uparrow, \quad h \in L_{\Pi}^{1} .
$$

Denote by $h_{l}$ the restriction of $h$ to the interval $5 l$. We say that $l$ is of type I if

$$
d^{\kappa-2} l^{2} \leq C\left\|h_{l}\right\|_{\Pi},
$$

where $C$ is a sufficiently large constant; otherwise we call $l$ an interval of type II. Clearly, we have

$$
\sum_{l \in \mathrm{I}} d^{\kappa-2} l^{2}<\infty
$$

and to get a contradiction we need to show

$$
\sum_{l \in \mathrm{II}} d^{\kappa-2} l^{2}<\infty
$$

Consider the 2D Hilbert transform

$$
H(z)=\int_{\mathbb{R}} \frac{h^{-}(t) d t}{(t-z)^{2}}, \quad\left(z \in \mathbf{C}_{+}\right) .
$$


where $h^{-}=\max \{0,-h\}$.

Lemma. If $l$ is of type II, then

$$
|H(z)| \gtrsim d^{\kappa}, \quad \forall z \in Q_{l}:=\{x+i y: x \in l, l<y<2 l\} .
$$

We prove this lemma in the next subsection, and we now explain how the lemma implies (3.6). Denote

$$
\psi=\sum_{l \in \mathrm{II}} d^{\kappa} l \cdot 1_{l}
$$

We have

$$
\sum_{l \in \mathrm{II}} d^{\kappa-2} l^{2} \asymp \int_{1}^{\infty} \frac{\psi(t) d t}{t^{2}}=\frac{8}{3} \int_{1}^{\infty} \frac{d A}{A^{3}} \int_{A / 2}^{A} \psi(t) d t .
$$

For every $A>1$ let

$$
H_{A}(z)=\int_{-C A}^{C A} \frac{h^{-}(t) d t}{(t-z)^{2}}
$$

where $C>0$ is a large constant, and let $\operatorname{II}(A)$ denote the set of all intervals $l \in \mathrm{II}$ intersecting $(A / 2, A)$. If $l \in \operatorname{II}(A)$ and $z \in Q_{l}$, then

$$
\left|H(z)-H_{A}(z)\right| \leq \int_{|t|>C A} \frac{h^{-}(t) d t}{|t-z|^{2}} \asymp \int_{|t|>C A} \frac{h^{-}(t) d t}{1+t^{2}} \ll 1,
$$

so by the lemma we have

$$
\left|H_{A}\right| \gtrsim A^{\kappa} \quad \text { on } \bigcup_{l \in \mathrm{II}(A)} Q_{l}
$$

Applying the weak- $L^{1}$ estimate for the 2D Hilbert transform, see [4], we get

$$
\sum_{l \in \mathrm{II}(A)} l^{2} \leq \operatorname{Area}\left(\left|H_{A}\right| \gtrsim A^{\kappa}\right) \lesssim A^{-\kappa} \int_{-C A}^{C A} h^{-}(t) d t,
$$

and therefore

$$
\int_{A / 2}^{A} \psi(t) d t \lesssim A^{\kappa} \sum_{l \in \mathrm{II}(A)} l^{2} \lesssim \int_{-C A}^{C A} h^{-}(t) d t,
$$

Combining this with (3.7), we conclude

$$
\sum_{l \in \mathrm{II}} d^{\kappa-2} l^{2} \lesssim \int_{1}^{\infty} \frac{d A}{A^{3}} \int_{-C A}^{C A} h^{-}(t) d t \lesssim\left\|h^{-}\right\|_{\Pi}
$$

which proves (3.6)

3.4. Proof of the lemma. Since $\alpha$ in the representation $\tilde{h}=\gamma+\alpha$ is increasing, we have

$$
\Delta_{l}^{*}[\tilde{h}] \gtrsim l d^{\kappa}
$$

On the other hand, for intervals of type II we have

$$
\Delta_{l}^{*}\left[\tilde{h}_{l}\right] \ll l d^{\kappa} .
$$

Indeed, if $\Delta_{l}^{*}\left[\tilde{h}_{l}\right] \gtrsim l d^{\kappa}$, then $\left|\tilde{h}_{l}\right| \gtrsim l d^{\kappa}$ on either $l^{\prime}$ or $l^{\prime \prime}$. Applying the weak type inequality with $A \asymp l d^{\kappa}$, we get

$$
d^{-2} l \lesssim \Pi\left\{\left|\tilde{h}_{l}\right|>A\right\} \lesssim A^{-1}\left\|h_{l}\right\|_{\Pi},
$$


which contradicts the definition of type II.

Denote $f \equiv f_{l}=1_{\mathbb{R} \backslash 5 l} \cdot h$, so $\tilde{h}=\tilde{h}_{l}+\tilde{f}_{l}$. From (3.8)-(3.9) we conclude that there are points $a \in l^{\prime}$ and $b \in l^{\prime \prime}$ such that

$$
\tilde{f}(b)-\tilde{f}(a) \geq \frac{c}{2} l d^{\kappa} .
$$

Represent $f=f^{+}-f^{-}$with $f^{+}=\max \{f, 0\}$, and note that the functions $\tilde{f}_{ \pm}:=\left(f^{ \pm}\right)^{\sim}$ are decreasing on $[a, b]$ :

$$
\tilde{f}_{ \pm}^{\prime}(x)=-\frac{1}{\pi} \int_{\mathbb{R} \backslash(5 l)} \frac{f^{ \pm}(t) d t}{(t-x)^{2}}<0, \quad(x \in 5 l) .
$$

From (3.10) it then follows that

$$
\tilde{f}_{-}(a)-\tilde{f}_{-}(b) \gtrsim l d^{\kappa},
$$

so there is a point $x_{*} \in(a, b)$ such that

$$
\frac{1}{\pi} \int \frac{f^{-}(t) d t}{\left(t-x_{*}\right)^{2}}=-\tilde{f}_{-}^{\prime}\left(x_{*}\right)=\frac{\tilde{f}_{-}(a)-\tilde{f}_{-}(b)}{b-a} \gtrsim d^{\kappa} .
$$

Observe that if $z \in Q_{l}$ and $t \in \mathbb{R} \backslash 5 l$, then

$$
\Re\left[\frac{1}{(t-z)^{2}}\right] \asymp \frac{1}{\left(t-x_{*}\right)^{2}},
$$

and we have

$$
\left|\int \frac{f^{-}(t) d t}{(t-z)^{2}}\right| \geq \Re \int \frac{f^{-}(t) d t}{(t-z)^{2}} \asymp \int \frac{f^{-}(t) d t}{\left(t-x_{*}\right)^{2}} \gtrsim d^{\kappa} .
$$

It follows that

$$
|H(z)| \geq\left|\int \frac{f_{l}^{-}(t) d t}{(t-z)^{2}}\right|-\left|\int \frac{h_{l}^{-}(t) d t}{(t-z)^{2}}\right| \gtrsim d^{\kappa},
$$

because

$$
\left|\int \frac{h_{l}^{-}(t) d t}{(t-z)^{2}}\right| \lesssim \frac{d^{2}}{l^{2}}\left\|h_{l}\right\|_{\Pi} \ll d^{\kappa}
$$

provided that the constant $C$ in (3.5) is large enough.

\section{Non-triviality of Toeplitz Kernels in Smirnov-Nevanlinna Class}

4.1. A version of the "little multiplier" theorem. In this section we prove the following statement. Let $\kappa \geq 0$ and suppose that $U=e^{i \gamma}, S=e^{i \sigma}$ satisfy conditions (1.4) of Theorem A.

Proposition. If $\gamma$ is almost $(\kappa)$ decreasing, then $N^{+}\left[U \bar{S}^{\epsilon}\right] \neq 0$ for all $\epsilon>0$.

Proof: By assumption we have

$$
\sum_{l \in \mathcal{B M}(\gamma)} d^{\kappa-2} l^{2}<\infty .
$$

Recall that the BM intervals of $\gamma$ are the components of the open set $\left\{\gamma^{*} \neq \gamma\right\}$, where $\gamma^{*}(x)=\max \gamma[x,+\infty)$. Denote

$$
f=\gamma^{*}-\gamma,
$$


so $f=0$ outside the union of BM intervals, and $f^{\prime}(x) \lesssim|x|^{\kappa}$ on BM intervals. By (4.1) we have $l \lesssim d$, and therefore

$$
0 \leq f \lesssim l d^{\kappa} \quad \text { on } \quad l,
$$

Together with (4.1) this implies

$$
f \in L_{\Pi}^{1} .
$$

The estimate (4.2) also shows that we can assume $l \geq d^{-\kappa}$ for all BM intervals; otherwise we can eliminate short intervals by adding a bounded function to $\gamma$ (this will not affect the $N^{+}$-kernel). In particular, we will assume that BM intervals don't cluster to a finite point.

The non-triviality of $N^{+}\left[U \bar{S}^{\epsilon}\right]$ is a consequence of the following statement which will be verified in the next two subsections.

Lemma. For any $\epsilon>0$, there is a function $\beta$ such that

$$
f+\beta \in \tilde{L}_{\Pi}^{1}, \quad \beta^{\prime}(x) \leq \epsilon|x|^{\kappa} \quad \text { for } \quad|x| \gg 1 .
$$

Indeed, if for instance $\sigma^{\prime}(x) \geq|x|^{\kappa}$ near $\pm \infty$, then we can write

$$
\gamma-\epsilon \sigma=-(f+\beta)+(\beta-\epsilon \sigma)+\gamma^{*} .
$$

The first term in the RHS is in $\tilde{L}_{\Pi}^{1}$, and the last two terms are decreasing near infinities, so we can apply the basic criterion (1.6).

4.2. Proof of the lemma. We will construct disjoint intervals $l_{n}$ such that they cover all $\mathrm{BM}$ intervals and satisfy the following two conditions:

$$
\sum_{n} d_{n}^{\kappa-2} l_{n}^{2}<\infty
$$

and

$$
\forall n \quad \exists \epsilon_{n} \in[0, \epsilon], \quad \int_{l_{n}} \frac{f(x)-\epsilon_{n}|x|^{\kappa} T_{n}(x)}{1+x^{2}} d x=0,
$$

where $T_{n}$ is the "tent" function of the interval $l_{n}$,

$$
T_{n}(x)=\operatorname{dist}\left(x, \mathbb{R} \backslash l_{n}\right) .
$$

Let us show that the existence of such intervals $l_{n}$ implies the statement of the lemma. Define

$$
\beta(x)=-\sum_{n} \epsilon_{n}|x|^{\kappa} T_{n}(x),
$$

and

$$
g(x)=f(x)-\sum \epsilon_{n}|x|^{\kappa} T_{n}(x) .
$$

Clearly, we have

$$
\left|\beta^{\prime}(x)\right| \lesssim \epsilon|x|^{\kappa},
$$

and all we need is to check $g \in \tilde{L}_{\Pi}^{1}$.

Let us show that $g$ belongs to the real Hardy space $\mathcal{H}_{\Pi}^{1}(\mathbb{R})$. We can represent $g$ as follows:

where

$$
g=\sum g_{n}=\sum \lambda_{n} \frac{g_{n}}{\lambda_{n}}:=\sum \lambda_{n} A_{n},
$$

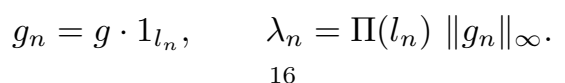


The functions $A_{n}=\lambda_{n}^{-1} g_{n}$ are "atoms":

$$
\int A_{n} d \Pi=\frac{1}{\lambda_{n}} \int_{l_{n}} g d \Pi=0 \quad \text { by } \quad(4.4),
$$

and

$$
\left\|A_{n}\right\|_{\infty}=\frac{\left\|g_{n}\right\|_{\infty}}{\lambda_{n}}=\frac{1}{\Pi\left(l_{n}\right)}
$$

Since

$$
\left\|g_{n}\right\|_{\infty} \lesssim d_{n}^{\kappa} l_{n}
$$

(use $l_{n} \lesssim d_{n}$ and (4.2) for the BM intervals covered by $l_{n}$ ), we have

$$
\sum \lambda_{n} \lesssim \sum \frac{l_{n}}{d_{n}^{2}} d_{n}^{\kappa} l_{n}<\infty \quad \text { by } \quad(4.3) .
$$

It follows that $\sum \lambda_{n} A_{n} \in \mathcal{H}_{\Pi}^{1}(\mathbb{R})$, see [5].

4.3. Construction of intervals $l_{n}$. Let us assume that all BM intervals $l$ lie in $[1,+\infty)$. In the general case we will need to apply the procedure described below to $\mathrm{BM}$ intervals in $(-\infty,-1]$ and in $[1,+\infty)$ separately.

We construct our intervals $l_{1}, l_{2}, \ldots$ by induction. The left endpoint $a_{1}$ of $l_{1}$ will be the left endpoint of the leftmost BM interval. Suppose the left endpoint $a_{n}$ of $l_{n}$ has been constructed so that $a_{n}$ is also the left endpoint of some BM interval $l=\left(a_{(l)}, b_{(l)}\right)$, i.e. $a_{n}=a_{(l)}$. Consider the function

$$
F(b)=\int_{a_{n}}^{b}\left[f-\epsilon|x|^{\kappa} T_{\left(a_{n}, b\right)}\right] d \Pi,
$$

where $T_{\left(a_{n}, b\right)}(\cdot)=\operatorname{dist}\left(\cdot,\left\{a_{n}, b\right\}\right)$ is the tent function. We define $b_{n}$, the right endpoint of $l_{n}$, as the nearest point in the complement of BM intervals at which $F$ is non-positive,

$$
b_{n}=\min \left\{b \geq b_{(l)}: f(b)=0, F(b) \leq 0\right\} .
$$

Since $f \in L_{\Pi}^{1}$, we have $F(+\infty)=-\infty$ and so $b_{n}<\infty$. Finally, we define $a_{n+1}$ as the leftmost endpoint of BM intervals not covered by $l_{1} \cup \cdots \cup l_{n}$. (Recall that we assumed that there are no finite cluster points.)

It is clear from the construction that the intervals $l_{n}$ cover all BM intervals. We also get (4.4) by defining $\epsilon_{n}$ from the equation

$$
\int_{a_{n}}^{b_{n}}\left[f-\epsilon_{n}|x|^{\kappa} T_{\left(a_{n}, b_{n}\right)}\right] d \Pi=0
$$

clearly we have $0<\epsilon_{n} \leq \epsilon$. In remains to verify (4.3). We have three types of intervals $l_{n}$ :

(a) $F\left(b_{n}\right)<0$ but there is a BM interval $l \subset l_{n}$ such that $|l| \asymp\left|l_{n}\right|$,

(b) $F\left(b_{n}\right)=0$,

(c) other intervals.

Property (4.3) is obvious for the collection of intervals of type (a): we have $l \ll d$ (except for finitely many l's) and therefore $d \asymp d_{n}$ and $d^{\kappa-2} l^{2} \asymp d_{n}^{\kappa-2} l_{n}^{2}$. 
To prove (4.3) for the collection of intervals of type (b), we note that $\epsilon_{n}=\epsilon$ if $l_{n} \in(\mathrm{b})$, and since

$$
\int_{a}^{b} x^{\kappa} T_{(a, b)}(x) \frac{d x}{x^{2}} \geq \frac{a^{\kappa}}{b^{2}} \int_{a}^{b} T_{(a, b)} \gtrsim \frac{a^{\kappa}}{b^{2}}(b-a)^{2},
$$

we have

$$
\sum_{(b)} \frac{d_{n}^{\kappa} l_{n}^{2}}{\left(d_{n}+l_{n}\right)^{2}} \lesssim \sum_{(b)} \int_{a_{n}}^{b_{n}}|x|^{\kappa} T_{\left(a_{n}, b_{n}\right)} d \Pi=\frac{1}{\epsilon} \int_{\bigcup_{(b)} l_{n}} f d \Pi<\infty .
$$

Since $d_{n} \rightarrow \infty$, it follows that there are only finitely many intervals $l_{n} \in(b)$ satisfying $d_{n} \leq l_{n}$, so the last estimate implies

$$
\sum_{(b)} d_{n}^{\kappa-2} l_{n}^{2}<\infty
$$

The argument for intervals of type (c) is the same if we can show that if $l_{n} \in(\mathrm{c})$, then $\epsilon_{n}>\epsilon / 2$, i.e.

$$
\int_{a_{n}}^{b_{n}}\left[f-\frac{\epsilon}{2}|x|^{\kappa} T_{\left(a_{n}, b_{n}\right)}\right] d \Pi>0 .
$$

Since $l_{n}$ is not of type (b), we have $F\left(b_{n}\right)<0$ and by construction, $b_{n}$ is the right endpoint of some BM interval $l=\left(c, b_{n}\right)$. Note that $|l| \ll\left|l_{n}\right|$ because $l_{n}$ is not of type (a). Since $f>0$ on $l$, we have

$$
\begin{gathered}
\int_{a_{n}}^{b_{n}}\left(f-\frac{\epsilon}{2} x^{\kappa} T_{\left(a_{n}, b_{n}\right)}\right) d \Pi>\int_{a_{n}}^{c}\left(f-\epsilon x^{\kappa} T_{\left(a_{n}, c\right)}\right) d \Pi+ \\
+\left[\epsilon \int_{a_{n}}^{c} x^{\kappa} T_{\left(a_{n}, c\right)} d \Pi-\frac{\epsilon}{2} \int_{a_{n}}^{b_{n}} x^{\kappa} T_{\left(a_{n}, b_{n}\right)} d \Pi\right] .
\end{gathered}
$$

The first term in the RHS is equal to $F(c)$ and therefore positive by construction. Since $|l| \ll\left|l_{n}\right|$, the second term in the RHS is also positive, and we get (4.5)

\section{Multiplier theOREM}

5.1. BM multipliers. Let $S$ be a unimodular function and let $0<p \leq \infty$. If $w \in L_{\Pi}^{1}$ is a real function, then we write

$$
w \in \mathcal{M}_{p}(S)
$$

if the outer function

$$
W=e^{w+i \tilde{w}}
$$

satisfies the following condition:

$$
\forall \epsilon>0, \quad \exists G \in N^{+}\left[\bar{S}^{\epsilon}\right], \quad W G \in H^{p} .
$$

In other words, $w \in \mathcal{M}_{p}(S)$ if the corresponding outer function belongs to $H^{p}$ up to an arbitrarily small (compared to $S$ ) factor.

We can restate this property in terms of Toeplitz kernels. 
Lemma. $w \in \mathcal{M}_{p}(S)$ iff

$$
\forall \epsilon>0, \quad N^{p}\left[\bar{S}^{\epsilon} \frac{\bar{W}}{W}\right] \neq 0 .
$$

Proof: $\Rightarrow$ Let $G \in N^{+}\left[\bar{S}^{\epsilon}\right]$ be such that $F:=G W \in H^{p}$. Then

$$
F \in N^{p}\left[\bar{S}^{\epsilon} \frac{\bar{W}}{W}\right]
$$

and the Toeplitz kernel is non-trivial. Indeed,

$$
\bar{S}^{\epsilon} \frac{\bar{W}}{W} F=\left(\bar{S}^{\epsilon} G\right) \bar{W} \in \mathcal{N}^{-} \cap L^{p}=\bar{H}^{p} .
$$

$\Leftarrow$ If $F$ is in the Toeplitz kernel, i.e. $F \in H^{p}$ and $F \bar{S}^{\epsilon} \bar{W} / W \in \bar{H}^{p}$, then we define $G=F / W \in \mathcal{N}^{+}$. Since

$$
\bar{S}^{\epsilon} G=\bar{S}^{\epsilon} \frac{\bar{W}}{W} F \frac{1}{\bar{W}} \in \mathcal{N}^{-},
$$

we have $G \in N^{+}\left[\bar{S}^{\epsilon}\right]$ and $W G \in H^{p}$.

Corollary. Suppose $(\arg S)^{\prime} \gtrsim|x|^{\kappa}$. If a real function $w_{0} \in L_{\Pi}^{1}$ satisfies the following condition:

$$
\forall \epsilon>0, \quad \exists w \in L_{\Pi}^{1}, \quad w \geq w_{0}, \quad \tilde{w}^{\prime}>-\epsilon|x|^{\kappa}+o\left(|x|^{\kappa}\right),
$$

then $w_{0} \in \mathcal{M}_{p}(S)$ for all $p<1$.

Proof: Without loss of generality, $(\arg S)^{\prime} \geq 2|x|^{\kappa}$ for $|x| \gg 1$. We have

$$
-\arg \left[\bar{S}^{2 \epsilon} \frac{\bar{W}_{0}}{W_{0}}\right]=2(\epsilon \arg S+\tilde{w})+2\left(\tilde{w}_{0}-\tilde{w}\right):=\alpha+\tilde{g}
$$

where

$$
\alpha^{\prime} \geq \epsilon|x|^{\kappa}, \quad(|x| \gg 1)
$$

and

$$
g \in L_{\Pi}^{1}, \quad g \leq 0 .
$$

For sufficiently large $N$, the function $\alpha+\arg b^{N}$, where $b$ is the Blaschke factor (1.9), is monotone increasing on $\mathbb{R}$, and therefore there is an inner function $\Phi$, not a finite Blaschke product, such that

$$
\alpha+\arg b^{N}=\arg \Phi+\delta, \quad\|\delta\|_{\infty} \leq \pi .
$$

Clearly, $N^{\infty}\left[e^{-i \tilde{g}}\right] \neq 0$, i.e.

$$
N^{\infty}\left[e^{i \delta} \bar{b}^{N} \Phi \bar{S}^{2 \epsilon} \frac{\bar{W}_{0}}{W_{0}}\right] \neq 0 .
$$

By (1.8), we also have

$$
N^{p}\left[e^{-i \delta} \bar{b}^{N}\right] \neq 0,
$$

provided that $p<1$ and $N$ is sufficiently large, and of course

$$
N^{\infty}\left[b^{2 N} \bar{\Phi}\right] \neq 0 .
$$

It follows that

$$
N^{p}\left[\bar{S}^{2 \epsilon} \frac{\bar{W}_{0}}{W_{0}}\right] \neq 0 .
$$


The main result of this section is the following version of the Beurling-Malliavin multiplier theorem.

Theorem. Suppose $(\arg S)^{\prime} \gtrsim|x|^{\kappa}$, and let $w_{0} \in L_{\Pi}^{1}$ be a real function. Then

$$
|x|^{-\frac{2+\kappa}{2}} w_{0}(x) \in \mathcal{D}(\mathbb{R}, \infty) \quad \Rightarrow \quad w_{0} \in \mathcal{M}_{p}(S), \quad(\forall p<1) .
$$

Here the notation $f \in \mathcal{D}(\mathbb{R}, \infty)$ means that there is a neighborhood of infinity where $f$ coincides with some function from the Dirichlet space $\mathcal{D}(\mathbb{R})$. Recall that the Hilbert space $\mathcal{D}(\mathbb{R})$ consists of functions $h \in L_{\Pi}^{1}$ such that the harmonic extension $u=u(z)$ of $h$ to $\mathbf{C}_{+}$has a finite gradient norm,

$$
\|h\|_{\mathcal{D}}^{2} \equiv\|u\|_{\nabla}^{2}=\int_{\mathbf{C}_{+}}|\nabla u|^{2} d A<\infty
$$

( $d A$ is the area measure). If $h \in \mathcal{D}(\mathbb{R})$ is a smooth function, then we also have

$$
\|h\|_{\mathcal{D}}^{2}=\int_{\mathbb{R}} \bar{h} \tilde{h}^{\prime} d x .
$$

In the next two subsections we use some ideas from the proof of Theorem 64 in [6].

5.2. Proof of the multiplier theorem. It is clear that we can assume that the function $w_{0}+i \tilde{w}_{0}$ is analytic and has a zero of sufficiently large multiplicity at the origin; in particular

$$
h_{0}(x):=|x|^{-\frac{2+\kappa}{2}} w_{0}(x) \in \mathcal{D}(\mathbb{R}) .
$$

Let us fix $\epsilon>0$. According to the last corollary we need to construct $w$ such that

$$
\text { (i) } w \in L_{\Pi}^{1}, \quad\left(\text { ii) } w \geq w_{0}, \quad(\text { iii }) \tilde{w}^{\prime}>-\epsilon|x|^{\kappa}+o\left(|x|^{\kappa}\right) .\right.
$$

We define

$$
w(x)=|x|^{\frac{2+\kappa}{2}} h(x)
$$

where $h$ is a solution of the following extremal problem:

$$
\min \left\{I(h): h \geq h_{0}\right\}, \quad I(h):=\|h\|_{\mathcal{D}}^{2}+\epsilon \int|x|^{\frac{2+\kappa}{2}}|h(x)| d \Pi(x) .
$$

The existence of a solution follows from the usual argument: the set

$$
\mathcal{A}=\left\{h:\|h\|_{\mathcal{D}} \leq I\left(h_{0}\right), \quad h \geq h_{0} \text { a.e. }\right\} \subset \mathcal{D}(\mathbb{R})
$$

is bounded, closed, and convex in $\mathcal{D}(\mathbb{R})$, therefore it is weakly compact. Let $I_{0}$ denote the minimum of $I(h)$ over $\mathcal{A}$. Then there is a sequence of functions $h_{n} \in \mathcal{A}$ such that $I\left(h_{n}\right) \rightarrow I_{0}$ and $h_{n}$ weakly converge to some function $g \in \mathcal{A}$. It is then routine to see that

$$
I_{0} \leq I(g) \leq \liminf I\left(h_{n}\right)=I_{0},
$$

so $g$ is a solution of the extremal problem.

By construction, $w$ satisfies (i) and (ii). To prove (iii) we first note that

$$
\tilde{h}^{\prime}(x) \geq-\epsilon|x|^{\frac{\kappa-2}{2}} \text {. }
$$

Indeed, by the extremality of $h$ we have

$$
\|\phi\|_{\mathcal{D}}^{2}+2 \int \phi \tilde{h}^{\prime}+\epsilon \int(|h+\phi|-|h|)(x) \frac{|x|^{\frac{2+\kappa}{2}}}{1+x^{2}} d x=I(h+\phi)-I(h) \geq 0
$$


for all smooth test functions $\phi=\phi(x) \geq 0$. (The integral $\int \phi \tilde{h}^{\prime}$ has to be interpreted in the sense of the theory of distributions.) Since

$$
\frac{\phi(x)}{x^{2}} \geq \frac{|h(x)+\phi(x)|-|h(x)|}{1+x^{2}}
$$

we conclude

$$
\|\phi\|_{\mathcal{D}}^{2}+2 \int \phi(x)\left[\tilde{h}^{\prime}(x)+\epsilon|x|^{\frac{\kappa-2}{2}}\right] d x \geq 0
$$

Replacing $\phi(x)$ with $\delta \phi(x)$ and letting $\delta \rightarrow 0$, we get

$$
\int \phi(x)\left[\tilde{h}^{\prime}(x)+\epsilon|x|^{\frac{\kappa-2}{2}}\right] d x \geq 0
$$

for all $\phi \geq 0$, which proves (5.1)

To derive (iii) from (5.1) we apply Lemma 5 in Section 2 with

$$
\alpha=1+\frac{\kappa}{2}, \quad \beta=\kappa, \quad \beta-\alpha=\frac{\kappa}{2}-1 .
$$

The parameters $\alpha$ and $\beta$ are admissible because for $\kappa \geq 2$ we have $\alpha \leq \beta$, and if $0 \leq \kappa \leq 2$ then $1 \leq \alpha \leq 2$ and $\alpha \leq \beta+1$.

\subsection{Multipliers and one-sided Lipschitz condition.}

Proposition. If $w \in L_{\Pi}^{1}, w \geq 0$, and $\tilde{w}^{\prime} \lesssim|x|^{\kappa}$, then

$$
|x|^{-\frac{2+\kappa}{2}} w(x) \in \mathcal{D}(\mathbb{R}, \infty) .
$$

Proof: We will assume that the function $w_{0}+i \tilde{w}_{0}$ is analytic and has a zero of sufficiently large multiplicity at the origin. Let $u=u(z)$ be the harmonic extention of $|x|^{-\frac{2+\kappa}{2}} w(x)$ to the upper half plane $\mathbf{C}_{+}$, and let $v=\tilde{u}$. We need to show that the gradient norm of $u+i v$ in $\mathbf{C}_{+}$is finite,

$$
\|u+i v\|_{\nabla}^{2}=\lim _{r \rightarrow \infty} \int_{\partial D(r)} u d v<\infty,
$$

where $D(r)$ is the semidisc $\{|z|<r\} \cap \mathbf{C}_{+}$.

We first prove that the integrals over $\partial D(r) \cap \mathbb{R}$ are uniformly bounded from above. Applying Lemma 4 in Section 2 with (admissible) parameters

$$
\alpha=1+\frac{\kappa}{2}, \quad \beta=\kappa, \quad \beta-\alpha=\frac{\kappa}{2}-1
$$

to the functions $f=w$ and $g=u$, we see that

$$
v^{\prime}(x) \lesssim|x|^{\frac{\kappa-2}{2}}, \quad x \in \mathbb{R} .
$$

Since $u>0$ we have

$$
\int_{\partial D(r) \cap \mathbb{R}} u d v=\int_{-r}^{r} v^{\prime} u \lesssim \int_{-r}^{r}|x|^{\frac{\kappa-2}{2}}|x|^{-\frac{2+\kappa}{2}} w(x) d x \lesssim\|w\|_{\Pi}<\infty .
$$

To finish the proof of the proposition it remains to show that the integrals

$$
\int_{\partial D(r) \backslash \mathbb{R}} u d v=r I^{\prime}(r), \quad I(r):=\frac{1}{2} \int_{0}^{\pi} u^{2}\left(r e^{i \theta}\right) d \theta,
$$


don't tend to $+\infty$ as $r \rightarrow \infty$. In fact, it is enough to show

$$
I(r) \nrightarrow \infty,
$$

because if $r I^{\prime}(r) \rightarrow+\infty$, then $I^{\prime}(r) \geq 1 / r$ for all $r \gg 1$, and we have $I(r) \rightarrow \infty$. Since $\kappa \geq 0$, we can apply the following lemma.

Lemma. If $u \in L^{1}\left(1+|x|^{-1}\right)$, then $I(r) \nrightarrow \rightarrow \infty$.

Proof: We will prove an equivalent statement for functions in the unit disc D. Let $f=u+i \tilde{u}$ be an analytic function in $\mathbf{D}$ such that

$$
\frac{u(\zeta)}{1-|\zeta|} \in L^{1}(\partial \mathbf{D})
$$

Define

$$
h(z)=\frac{1+z}{1-z} u(z), \quad z \in \mathbf{D}
$$

and denote by $h^{*}(\zeta), \zeta \in \partial \mathbf{D}$, the angular maximal function. By Hardy-Littlewood maximal theorem,

$$
h^{*} \in L_{\text {weak }}^{1}(\partial \mathbf{D})
$$

Let us show that as $\epsilon \rightarrow 0$,

$$
\frac{1}{\epsilon} \int_{C_{\epsilon}}|f(z)|^{2}|d z| \not \rightarrow \infty, \quad C_{\epsilon}:=\{|1-z|=\epsilon\} \cap \mathbf{D} .
$$

We have

$$
\frac{1}{\epsilon} \int_{C_{\epsilon}}|f|^{2}=\epsilon \int_{C_{\epsilon}}|h|^{2} \lesssim\left[\epsilon h^{*}(\zeta)\right]^{2}+\left[\epsilon h^{*}(\bar{\zeta})\right]^{2},
$$

where $\zeta \in \partial \mathbf{D},|1-\zeta|=\epsilon$. The RHS can not tend to infinity because otherwise for all small $\epsilon$, we would have

$$
h^{*}(\zeta)+h^{*}(\bar{\zeta}) \gg \frac{1}{\epsilon}
$$

on an interval of length $\epsilon$, which would contradict (5.2).

\subsection{A version of BM1.}

Proposition. Suppose $(\arg S)^{\prime} \gtrsim|x|^{\kappa}$ and let $\Theta$ be a meromorphic inner function satisfying $\left|\Theta^{\prime}\right| \lesssim|x|^{\kappa}$. Then

$$
W \in N^{+}[\bar{\Theta}] \quad \Rightarrow \quad \log |W| \in \mathcal{M}_{p}(S), \quad(\forall p<1) .
$$

Proof: We have $W \bar{\Theta}=\bar{H}$ for some $H \in \mathcal{N}^{+}$. Define

$$
W_{1}=W H+\Theta,
$$

and let $W_{1}^{e}$ be the outer part of $W_{1}$. From the identity

$$
\bar{\Theta}^{2} W_{1}=\bar{\Theta} W \bar{\Theta} H+\bar{\Theta}=\bar{H} \bar{W}+\bar{\Theta}=\bar{W}_{1},
$$

we deduce

and

$$
\left|W_{1}\right|=|W \bar{W} \Theta+\Theta|=1+|W|^{2} \geq 1,
$$

$$
\left|W_{1}^{e}\right| \geq 1, \quad|W| \leq\left|W_{22}^{e}\right|, \quad\left(\arg W_{1}^{e}\right)^{\prime} \lesssim|x|^{\kappa} .
$$


By Proposition 5.3 and the multiplier theorem, we have $\log \left|W_{1}\right| \in \mathcal{M}_{p}(S)$ and therefore

$$
\log |W| \in \mathcal{M}_{p}(S)
$$

Corollary. Let $S$ and $\Theta$ be as above. Then for any meromorphic inner function $J$ and any $p<1$, we have

$$
N^{+}[\bar{\Theta} J] \neq 0 \quad \Rightarrow \quad \forall \epsilon, \quad N^{p}\left[\bar{S}^{\epsilon} \bar{\Theta} J\right] \neq 0 .
$$

Proof: Take an outer function $W \in N^{+}[\bar{\Theta} J]$. Then $W \in N^{+}[\bar{\Theta}]$, and by the last proposition,

$$
\exists G \in N^{+}\left[\bar{S}^{\epsilon}\right] . \quad W G \in H^{p} .
$$

It then follows that

$$
W G \in N^{+}\left[\bar{S}^{\epsilon} \bar{\Theta} J\right] \cap H^{p}=N^{p}\left[\bar{S}^{\epsilon} \bar{\Theta} J\right] .
$$

\section{Non-triviality of Toeplitz kernels in Hardy spaCeS}

In this final section we finish the proof of Theorems A and B.

6.1. Approximation by inner functions. It is well known that given any two intertwining discrete sets $A=\left\{a_{n}\right\}$ and $B=\left\{b_{n}\right\}$ of real numbers, $\ldots a_{n}<b_{n}<a_{n+1} \ldots$, there exists a meromorphic inner function $\Theta$ such that

$$
\{\Theta=1\}=A, \quad\{\Theta=-1\}=B .
$$

Indeed, the sequences $A, B$ determine the set

$$
E=\{\Im \Theta>0\} \cap \mathbb{R}=\cup\left(a_{n}, b_{n}\right),
$$

and we can define $\Theta$ in $\mathbf{C}_{+}$by the (Krein's shift) formula

$$
\frac{1}{\pi i} \log \frac{\Theta+1}{\Theta-1}=\mathcal{S} u+i c, \quad u:=1_{E}-\frac{1}{2}, \quad c \in \mathbb{R},
$$

where $\mathcal{S} u$ is the Schwarz integral (1.5), so $\Re[\mathcal{S} u]$ is the Poisson extension of $u$ to the halfplane. (Note that $u$ is the boundary function of the expression in the LHS of (6.2), provided that $\Theta$ is an inner function with level sets $A$ and $B$, and in fact Krein's shift formula parametrizes all such inner functions.)

An immediate consequence of this construction is the following statement: for any increasing, continuous function $\sigma: \mathbb{R} \rightarrow \mathbb{R}$, there exists a meromorphic inner function $\Theta=e^{i \theta}$ such that

$$
\|\theta-\sigma\|_{\infty} \leq \pi .
$$

We will need the following version of this statement.

Lemma. If $\sigma^{\prime}(x) \asymp|x|^{\kappa}$, then there is a meromorphic inner function $\Theta=e^{i \theta}$ such that

$$
\theta-\sigma \in L^{\infty}(\mathbb{R}), \quad \theta^{\prime}(x) \asymp|x|^{\kappa} .
$$


Proof: We can assume that $\sigma$ is strictly increasing on $\mathbb{R}$. Define the intertwining sequences $A=\left\{a_{n}\right\}$ and $B=\left\{b_{n}\right\}$ by the equations

$$
\sigma\left(a_{n}\right)=2 \pi n, \quad b_{n}=\frac{a_{n}+a_{n+1}}{2}, \quad(n \in \mathbf{Z}),
$$

so we have

$$
a_{n} \asymp(\operatorname{sign} n)|n|^{\frac{1}{1+\kappa}},
$$

and

$$
\delta_{n}:=b_{n}-a_{n} \asymp\left|a_{n}\right|^{-\kappa} .
$$

Let $\Theta$ be an inner function satisfying (6.1),

$$
\|\theta-\sigma\|_{\infty} \leq 2 \pi
$$

and let $\mu_{1}, \mu_{-1}$ be the corresponding (Aleksandrov-Clark's) measures defined by the Herglotz representation

$$
\frac{1+\Theta}{1-\Theta}=\mathcal{S} \mu_{1}+\text { const }, \quad \frac{1-\Theta}{1+\Theta}=\mathcal{S} \mu_{-1}+\text { const. }
$$

The measures $\mu_{1}, \mu_{-1}$ have the following form:

$$
\mu_{1}=\sum \alpha_{n} \delta_{a_{n}}, \quad \mu_{-1}=\sum \beta_{n} \delta_{b_{n}}
$$

for some positive numbers $\alpha_{n}, \beta_{n}$. (It is easy to see that $\mu_{ \pm 1}\{\infty\}=0$ though we don't actually need this fact.) We claim that

$$
\alpha_{n} \asymp \delta_{n}, \quad \beta_{n} \asymp \delta_{n} .
$$

The estimate $\theta^{\prime}(x) \asymp|x|^{\kappa}$ easily follows from (6.3). Since

$$
\left|\Theta^{\prime}\right| \asymp\left|1-\Theta^{2}\right|\left|\left(\mathcal{S} \mu_{1}\right)^{\prime}\right|, \quad\left|\Theta^{\prime}\right| \asymp\left|1+\Theta^{2}\right|\left|\left(\mathcal{S} \mu_{-1}\right)^{\prime}\right|,
$$

we have

$$
\theta^{\prime}(x) \asymp \min \left\{\sum \frac{\alpha_{n}}{\left(x-a_{n}\right)^{2}}, \sum \frac{\beta_{n}}{\left(x-b_{n}\right)^{2}}\right\}, \quad(x \in \mathbb{R}) .
$$

It follows that if $x \in\left(a_{m}, a_{m+1}\right)$, then by (6.3)

$$
\theta^{\prime}(x) \asymp \int_{|t-x| \gtrsim \delta_{m}} \frac{d t}{(x-t)^{2}} \asymp \delta_{m}^{-1} \asymp\left|a_{m}\right|^{\kappa} \asymp|x|^{\kappa} .
$$

Proof of (6.3). We will explain the estimate for $\alpha_{n}$ 's; the proof for $\beta_{n}$ 's is similar. According to (6.2), we have

$$
\frac{1-\Theta}{1+\Theta}=\text { const } e^{K u},
$$

where $u=1_{E}-1 / 2$,

$$
E=\bigcup_{k=-\infty}^{\infty}\left(a_{k}, b_{k}\right)
$$

and $K u$ is the improper integral

$$
K u(z)=\int \frac{u(t) d t}{t-z}, \quad\left(z \in \mathbf{C}_{+}\right) .
$$

By construction,

$$
\alpha_{n}=\text { const } \operatorname{Res}_{a_{n}} e^{K u} .
$$


Denote

$$
g_{n}(z)=\exp \left\{\int_{b_{n-1}}^{b_{n}} \frac{u(t) d t}{t-z}\right\}=\frac{\sqrt{\left(b_{n}-z\right)\left(b_{n-1}-z\right)}}{a_{n}-z}
$$

and

$$
A_{n}=\exp \left\{\int_{\mathbb{R} \backslash\left(b_{n-1}, b_{n}\right)} \frac{u(t) d t}{t-a_{n}}\right\},
$$

SO

$$
\operatorname{Res}_{a_{n}} e^{K u}=A_{n} \operatorname{Res}_{a_{n}} g_{n}, \quad\left|\operatorname{Res}_{a_{n}} g_{n}\right| \asymp \delta_{n} .
$$

It remains to show that $A_{n}=e^{O(1)}$. This can be done as follows.

For $j>n$ we have

$$
\begin{aligned}
\int_{a_{j}}^{a_{j+1}} \frac{u(t) d t}{t-a_{n}} & =\log \frac{b_{j}-a_{n}}{a_{j}-a_{n}}-\log \frac{a_{j+1}-a_{n}}{b_{j}-a_{n}} \\
& =\log \left(1+\frac{\delta_{j}}{a_{j}-a_{n}}\right)-\log \left(1+\frac{\delta_{j}}{b_{j}-a_{n}}\right) \\
& =\frac{\delta_{j}}{a_{j}-a_{n}}-\frac{\delta_{j}}{b_{j}-a_{n}}+O\left(\frac{\delta_{j}^{2}}{\left(a_{j}-a_{n}\right)^{2}}\right)=O\left(\frac{\delta_{j}^{2}}{\left(a_{j}-a_{n}\right)^{2}}\right),
\end{aligned}
$$

where we used the relation $\log (1+x)=x+O\left(x^{2}\right)$ for $0<x \lesssim 1$. Since

$$
\begin{aligned}
\sum_{j=n+1}^{\infty} \frac{\delta_{j}^{2}}{\left(a_{j}-a_{n}\right)^{2}} & \asymp \sum_{j=n+1}^{\infty} \frac{\delta_{j}}{a_{j}^{\kappa}\left(a_{j}-a_{n}\right)^{2}} \\
& \asymp \int_{a_{n}+\delta_{n}}^{\infty} \frac{d t}{t^{\kappa}\left(t-a_{n}\right)^{2}}=\int_{a_{n}+\delta_{n}}^{2 a_{n}}+\int_{2 a_{n}}^{\infty} \\
& \lesssim \frac{1}{a_{n}^{\kappa}} \int_{a_{n}+\delta_{n}}^{\infty} \frac{d t}{\left(t-a_{n}\right)^{2}}+\int_{a_{n}}^{\infty} \frac{d t}{t^{2+\kappa}} \\
& \asymp \frac{1}{a_{n}^{\kappa}} \frac{1}{\delta_{n}}+\frac{1}{a_{n}^{1+\kappa}}=O(1),
\end{aligned}
$$

we get

$$
\int_{b_{n}}^{\infty} \frac{u(t) d t}{t-a_{n}}=O(1)
$$

A similar estimate holds for the integral over $\left(-\infty, b_{n-1}\right)$, and we have $A_{n}=e^{O(1)}$.

6.2. Proof of Theorem A. The first part of the theorem was established in Section 3. The second part states that if $\gamma$ is almost decreasing and $\epsilon>0$, then

$$
N^{p}\left[U \bar{S}^{2 \epsilon}\right] \neq 0, \quad(p<1 / 3) .
$$

By Lemma 6.1 there exists an inner function $\Theta$ satisfying

$$
(\arg \Theta)^{\prime} \asymp|x|^{\kappa} \text {. }
$$

We will assume that $U^{2} \Theta$ has an increasing argument (otherwise we can replace $\Theta$ with $\Theta^{n}$ for a large integer $n$ ). We will also assume that $S \bar{\Theta}$ has an increasing, 
unbounded argument (otherwise we replace $S$ with a large power). By Proposition 4.1 we have

$$
N^{+}\left[U \Theta^{1-\epsilon} \bar{\Theta}\right] \neq 0
$$

Since the argument of $U \Theta^{1-\epsilon}$ is increasing, there is an inner function $J$ such that

$$
U \Theta^{1-\epsilon}=X J, \quad\|\arg X\|_{\infty} \leq \pi .
$$

From (6.5) we have $N^{+}[J \bar{\Theta}] \neq 0$, and so by Corollary 5.4

$$
N^{p}\left[J \bar{\Theta} \bar{S}^{\epsilon}\right] \neq 0, \quad(p<1) .
$$

Note that

$$
U \bar{S}^{2 \epsilon}=\left(U \Theta^{1-\epsilon} \bar{\Theta} \bar{S}^{\epsilon}\right)\left(\Theta^{\epsilon} \bar{S}^{\epsilon}\right)=X \quad\left(J \bar{\Theta} \bar{S}^{\epsilon}\right)\left(\Theta^{\epsilon} \bar{S}^{\epsilon}\right) .
$$

Since the argument of $S^{\epsilon} \bar{\Theta}^{\epsilon}$ is increasing and unbounded, we can find an infinite Blaschke product $\Psi$ such that

$$
\Theta^{\epsilon} \bar{S}^{\epsilon}=Y \bar{\Psi}, \quad\|\arg Y\|_{\infty} \leq \pi .
$$

Thus the symbol $U \bar{S}^{2 \epsilon}$ has the following representation:

$$
U \bar{S}^{2 \epsilon}=\left(J \bar{\Theta} \bar{S}^{\epsilon}\right)(X Y \bar{\Psi}), \quad\|\arg X Y\|_{\infty} \leq 2 \pi,
$$

and by (1.8) we have

$$
N^{p}[X Y \bar{\Psi}] \neq 0, \quad(p<1 / 2)
$$

Combining (6.6) and (6.7), we get (6.4) by Hölder's inequality.

Remark. It is clear from the proof that if $\gamma$ is almost decreasing, then the $N^{p}$-kernels are infinite dimensional.

6.3. Proof of Theorem B. Recall that $J$ is a meromorphic inner function, $S=e^{i \sigma}$ with $\sigma^{\prime}(x) \asymp|x|^{\kappa}$, and $c=c(J, S ; \kappa)$. Applying Theorem A (or rather its corollary) to $U=J$ we conclude that if $a<c$ then $N^{+}\left[J \bar{S}^{a}\right]=0$ and therefore $N^{p}\left[J \bar{S}^{a}\right]=0$ for all $p>0$. On the other hand, if $a>c$, then $N^{p}\left[J \bar{S}^{a}\right] \neq 0$ for some $p>0$, and in fact the kernel is infinite dimensional, as we just mentioned. The following proposition completes the proof.

A unimodular function $S$ is called tempered if $\exists n, S^{\prime}(x)=O\left(|x|^{n}\right)$ as $x \rightarrow \infty$.

Proposition. If $S$ is a tempered unimodular function, then for any meromorphic inner function $J$ and any $p>0$,

$$
\operatorname{dim} N^{p}[J \bar{S}]=\infty \quad \Rightarrow \quad \operatorname{dim} N^{\infty}[J \bar{S}]=\infty .
$$

Proof: First of all we observe that the statement is true if $S$ is a tempered inner function, $S=\Theta$. By Carleson's type embedding theorem [34], all elements in $N^{p}[J \bar{\Theta}]$ have at most polynomial growth at infinity, see details in [23], Section 4.1. Since the kernel is infinite dimensional, it contains functions with many zeros in $\mathbf{C}_{+}$. Dividing such functions by appropriate polynomials we obtain functions in $N^{\infty}[J \bar{\Theta}]$.

Let now $S$ be an arbitrary tempered unimodular function. By Lemma 6.1 we can find a tempered inner function $\Theta$ and a bounded real-valued function $\chi$ such that

$$
S=\Theta \bar{X},{ }_{26} \quad X=e^{2 i \chi} .
$$


By the previous observation, we have

$$
\operatorname{dim} N^{\infty}[J \bar{\Theta}]=\infty,
$$

and it remains to show that

$$
\exists n, \quad N^{\infty}\left[X \bar{b}^{n}\right] \neq 0,
$$

( $b$ is the Blaschke factor (1.9)). Indeed, combining (6.8) and (6.9) we conclude that the kernel

$$
N^{\infty}\left[J \bar{S} \bar{b}^{n}\right]=N^{\infty}\left[J \bar{\Theta} X \bar{b}^{n}\right]
$$

is infinite dimensional, which allows us to get rid of $b^{n}$.

To prove (6.9), consider the outer function

$$
H=e^{\tilde{\chi}-i \chi}, \quad \text { so } \quad X=\frac{\bar{H}}{H} .
$$

We will have

$$
(z+i)^{-n} H(z) \in N^{\infty}\left[X \bar{b}^{n}\right], \quad(n \gg 1)
$$

if we can show that $h:=|H|=e^{\tilde{\chi}}$ has at most polynomial growth at infinity. Without loss of generality, we can assume that the $L^{\infty}$-norm of $\chi$ is so small that $h \in L_{\Pi}^{2}$. We have

$$
h(x)-h(0) \leq \int_{0}^{x}\left|h^{\prime}\right|=\int_{0}^{x} h\left|\tilde{\chi}^{\prime}\right| \lesssim\|h\|_{L_{\Pi}^{2}}\left(1+x^{2}\right)^{1 / 2}\left(\int_{0}^{x}\left|\tilde{\chi}^{\prime}\right|^{2}\right)^{1 / 2} .
$$

Since $\left|\chi^{\prime}(t)\right| \lesssim|t|^{n}$ by construction, for each $x>0$ we can represent $\chi$ as the sum of two smooth functions,

$$
\chi=\chi_{1}+\chi_{2},
$$

such that

$$
\left\|\chi_{1}^{\prime}\right\|_{L^{2}} \lesssim|x|^{n}, \quad\left\|\chi_{2}\right\|_{L^{\infty}} \asymp 1, \quad \chi_{2}=0 \quad \text { on } \quad(-2 x, 2 x) .
$$

(For example, take $\chi_{1}=\phi \chi$, where $\phi$ is a smooth "bump" function such that $\phi$ is equal to 1 on $(-2 x, 2 x)$ and 0 on $\mathbb{R} \backslash(-3 x, 3 x)$.) Then we have

$$
\left\|\tilde{\chi}_{1}^{\prime}\right\|_{L^{2}} \lesssim|x|^{n}, \quad\left|\tilde{\chi}_{2}^{\prime}\right| \lesssim 1 \quad \text { on }(0, x),
$$

and so (6.10) shows that $h$ has at most polynomial growth.

\section{REFERENCES}

[1] Beurling, A., Malliavin, P. On Fourier transforms of measures with compact support, Acta Math. 107 (1962), 291-302

[2] Beurling, A., Malliavin, P. On the closure of characters and the zeros of entire functions, Acta Math. 118 (1967), 79-93

[3] Bruna J., Olevskit, A., Ulanovskit, A. Completeness in $L^{1}(\mathbb{R})$ of discrete translates and related questions for quasi-analytic classes, Rev. Mat. Iberoamericana 22 (2005), 1-16

[4] Calderón, A., Zygmund, A. On the existence of certain singular integrals, Acta Math. 88 (1952), 85-139

[5] Coifman, R., Weiss, G. Extensions of Hardy spaces and their use in analysis, Bull. AMS 83 (1977), 569-645

[6] De Branges, L. Hilbert spaces of entire functions. Prentice-Hall, Englewood Cliffs, NJ, 1968

[7] Garnett, J. Bounded analytic functions. Academic Press, New York, 1981

[8] Gohberg, I., Krein, M. Theory and applications of Volterra operators in Hilbert space. AMS, Providence, RI, 1970 
[9] Havin, V., JöRICKE, B. The uncertainty principle in harmonic analysis. Springer-Verlag, Berlin, 1994.

[10] Havin, V., Mashreghi, J. Admissible majorants for model subspaces of $H^{2}$; I. Slow winding of the generating inner function, II. Fast winding of the generating inner function, Canad. J. Math. 55 (2003), 1231-1263, 1264-1301.

[11] Higgins, J. Completeness and basis properties of sets of special functions. Cambridge Univ. Press, Cambridge, 1977

[12] Hruschev S., Nikolskit, N., Pavlov, B. Unconditional bases of exponentials and of reproducing kernels, Lecture Notes in Math., Vol. 864, 214-335

[13] Hunt R., Muckenhoupt B., Wheeden R. Weighted norm inequalities for the conjugate functions and Hilbert transform, Trans. AMS 176 (1973), 227-251

[14] Kahane, J.-P. Travaux de Beurling et Malliavin, Seminaire Bourbaki. Exposés 223 á 228, 1962

[15] Khabibullin, B. Completeness of exponential systems and uniqueness sets. Bashkir State Univ. Press, Ufa, 2006

[16] Koosis, P. Introduction to $H^{p}$ spaces. Cambridge Univ. Press, Cambridge, 1980

[17] Koosis, P. The logarithmic integral, Vol. I \& II. Cambridge Univ. Press, Cambridge, 1988

[18] Koosis, P. Lecons sur le Theorem de Beurling et Malliavin. Les Publications CRM, Montreal, 1996

[19] Koosis, P. Kargaev's proof of Beurling's lemma (unpublished manuscript)

[20] Levin, B. Distribution of zeros of entire functions. AMS, Providence, RI, 1980

[21] Levinson, N. Gap and density theorems. AMS, New York, 1940

[22] Lyubarskit, Ju., Seip, K. Complete interpolating sequences for Paley-Wiener spaces and Muckenhoupt's $\left(A_{p}\right)$ condition, Rev. Mat. Iberoamericana 13 (1997), 361-376

[23] Makarov, N., Poltoratski, A. Meromorphic inner functions, Toeplitz kernels, and the uncertainty principle, in Perspectives in Analysis, Springer Verlag, Berlin, 2005, 185-252

[24] Mashreghi, J., Nazarov, F., Havin, V. Beurling-Malliavin multiplier theorem: the seventh proof, St. Petersburg Math. J. 17 (2006), 699-744

[25] Nazarov, F. The Beurling lemma via the Bellman function (unpublished manuscript)

[26] NikolskiI, N.K. Bases of exponentials and the values of reproducing kernels, Dokl. Acad. Nauk SSSR 252, (1980), 1316-1320

[27] Nikolskit, N. Operators, functions, and systems: an easy reading, Vol. I \& II. AMS, Providence, RI, 2002

[28] Ortega-Cedrá, J., Seip, K. Fourier frames, Annals of Math. 155 (2002), 789-806

[29] Paley, R., Wiener, N. Fourier transform in the complex domains. AMS, New York, 1934

[30] PAVlov, B. The basis property of a system of exponentials and the condition of Muckenhoupt, Dokl. Acad. Nauk SSSR 247, (1979), 37-40

[31] Redheffer, R. Completeness of sets of complex exponentials, Advances in Math. 24, 1977, $1-62$

[32] Schwartz, L. Études des sommes d'exponentielles réelles. Hermann, Paris, 1943

[33] SEIP, K. Interpolation and sampling in spaces of analytic functions, AMS, 2004

[34] Treil S., Volberg, A. Embedding theorems for invariant subspaces of inverse shift, Proceedings of LOMI Seminars, 149 (1986), 38-51

California Institute of Technology, Department of Mathematics, Pasadena, CA 91125, USA

E-mail address: makarov@its.caltech.edu

Texas A\& M University, Department of Mathematics, College Station, TX 77843, USA

E-mail address: alexeip@math.tamu.edu 